CAHIERS DE

NARRATOLOGIE

\section{Cahiers de Narratologie}

Analyse et théorie narratives

$36 \mid 2019$

Rhétorique et représentations de la culture mafieuse. Images, rituels, mythes et symboles

\title{
Sémiotique judiciaire : crime et signe
}

\section{Marcel Danesi}

Translator. Manuela Bertone (de l'italien)

\section{(2) OpenEdition \\ Journals}

Electronic version

URL: http://journals.openedition.org/narratologie/10080

ISSN: 1765-307X

Publisher

LIRCES

Electronic reference

Marcel Danesi, « Sémiotique judiciaire : crime et signe », Cahiers de Narratologie [Online], 36 | 2019,

Online since 20 December 2019, connection on 20 December 2019. URL : http://

journals.openedition.org/narratologie/10080

This text was automatically generated on 20 December 2019.

Article L.111-1 du Code de la propriété intellectuelle. 


\title{
Sémiotique judiciaire : crime et signe
}

\author{
Marcel Danesi
}

Translation : Manuela Bertone (de l'italien)

\section{Introduction}

La criminologie en tant que science judiciaire s'est diffusée en Angleterre à peu près en même temps que la littérature gothique et policière, à la fin du XIXe siècle ${ }^{1}$. Même si les autopsies et autres techniques d'enquête remontent à la chine médiévale et à l'Angleterre du XII e siècle, c'est la création du Bureau du Coroner, chargé de tenir les dossiers sur toutes les affaires pénales et de mener des enquêtes sur des meurtres et décès suspects, qui marque un tournant. Les colons britanniques exportent le système du Coroner en Amérique, au Massachusetts où, à partir de 1877, il devient le Bureau du Médecin légiste. À la même époque, de nouvelles techniques de résolution d'affaires criminelles font leur apparition en Europe et aux Etats-Unis, permettant aux enquêtes d'être de plus en plus encadrées par la science. En 1829 déjà, le Metropolitan Police Act (loi sur la police métropolitaine) inaugure un service de détectives à Scotland Yard chargé d'enquêter sur les crimes à l'aide de techniques nouvelles comme la prise d'empreintes digitales. Vers la fin du XIX siècle, l'étude de l'esprit criminel fait également son apparition, qui préfigure la psychologie judiciaire. En 1876, par exemple, le médecin italien Cesare Lombroso examine la morphologie de criminels connus, en classant notamment les traits d'expression faciale, la taille du crâne et la structure de l'œil: il arrive à la conclusion que les criminels se différencient du reste de la population par des traits spécifiques. Bien que les idées de Lombroso soient aujourd'hui largement contestées, son approche a jeté les bases de la science moderne du profilage criminel, qui repose sur le principe que, si les criminels ne se ressemblent pas, ils sont susceptibles d'agir et de se comporter de la même manière.

Comme nous l'avons dit, ces développements vont de pair avec l'essor et la diffusion de la littérature gothique et de la littérature policière. Bon nombre des techniques, des 
idées et des pratiques utilisées dans les enquêtes criminelles du XIX ${ }^{e}$ siècle s'inspirent en fait des démarches propres aux détectives fictifs comme C. Auguste Dupin (créé par Edgar Allan Poe) et Sherlock Holmes (créé par Arthur Conan Doyle). Comme Ronald Thomas l'a soutenu et abondamment illustré ${ }^{2}$, les premières méthodes et les premiers appareils de la criminologie (tels le polygraphe, la photo d'identité, les empreintes digitales ou l'analyse photographique) dérivent de la fiction policière ${ }^{3}$. Réciproquement, de nouvelles techniques médico-légales sont rapidement adoptées et adaptées par les auteurs de fiction afin de rendre les histoires plus passionnantes, plus réalistes et conformes au scientisme culturel ambiant. L'interaction entre science médico-légale et roman policier nourrit une fascination pour le crime en tant que tel, qui persiste toujours, comme en témoigne le grand nombre de récits criminels au cinéma, à la télévision et dans d'autres médias. Au moment où la science médico-légale et le roman policier tissent ces premiers liens, s'affirme la sémiotique de Peirce ${ }^{4}$, fondée sur un substrat intellectuel similaire. Peirce saisit en effet l'importance de la fiction policière comme modèle pour l'analyse sémiotique et pour la compréhension de la cognition humaine. Élucider un meurtre, par exemple, revient à déchiffrer les signes associés à sa perpétration et, partant, leur lien avec l'esprit de l'auteur et le milieu culturel dans lequel le crime a été commis. Dans des travaux récents, nous avons appelé sémiotique judiciaire l'étude de ce lien ${ }^{5}$ et mis en évidence la continuité historique entre la fiction policière, les sciences judiciaires et la sémiotique de Peirce.

Notre article part du principe que la sémiotique judiciaire est opératoire pour tous les aspects de l'étude de la criminalité. L'objectif est de montrer et démontrer tant aux sémioticiens qu'aux médecins légistes que le crime est un terrain fertile pour l'analyse sémiotique. Celle-ci peut fournir des informations clés non seulement sur les dimensions culturelles du délit, mais également sur le type de sémiose qui caractérise les actes criminels et, par voie de conséquence, sur la présence du crime dans les sociétés humaines. Nous considérons que la sémiotique judiciaire serait en mesure d'améliorer et d'élargir considérablement le domaine de la recherche médico-légale et de la criminologie.

\section{La sémiotique et le roman policier}

Dans un ouvrage bien connu désormais, Dupin, Holmes, Peirce: The Sign of Three, édité par Umberto Eco et Thomas A. Sebeok ${ }^{6}$, la criminologie moderne et la médicine légale sont associées, à la fois implicitement et explicitement, aux romans policiers d'Edgar Allan Poe et d'Arthur Conan Doyle. D'une certaine manière, il s'agit là du texte fondateur de ce que nous avons appelé la sémiotique judiciaire. Les historiens de la littérature considèrent trois récits de Poe, Double assassinat dans la rue Morgue (1841), Le Mystère de Marie Roget (1842) et La Lettre volée (1844) comme les premiers exemples de polar moderne. Dans ces récits, Poe crée le personnage du détective protagoniste, C. Auguste Dupin, héros intellectuel qui résout les crimes en faisant preuve d'un brillant raisonnement et d'une habile analyse sémiotique. Peirce a été intrigué par l'adresse de Dupin - qui fait preuve de fulgurantes intuitions en interprétant les indices laissés par le criminel - au point de la qualifier d'abduction, comme chacun sait.

En fait, Dupin utilise différents types de raisonnement à diverses étapes de ses enquêtes. Il applique le raisonnement déductif pour l'organisation des indices, un peu comme les pièces d'un puzzle; il utilise le raisonnement inductif pour obtenir une 
évaluation de l'image qui se dessine et utilise enfin l'abduction pour interpréter cette image, révélant en fin de compte la véritable histoire qui se cache derrière le crime.

Holmes, le successeur de Dupin, emploie exactement le même genre de raisonnement tripartite. Dans la première histoire de Holmes, Une étude en rouge (1867), son assistant le docteur Watson, compare le grand détective à Dupin, rendant ainsi un hommage indirect à Poe, le père fondateur du roman policier. Ce dernier se dessine en un certain sens comme une comédie de mœurs moderne. Le mal doit être identifié et vaincu, non pas avec la violence ou manu militari, mais par le truchement des stratégies de la pensée. À l'époque médiévale, le mal était vaincu par les forces spirituelles; aujourd'hui, il est vaincu par un détective ou un super-héros qui se bat contre le crime en utilisant les pouvoirs de la raison. Dramatisant des événements bibliques, la comédie de mœurs fleurit au XVe et au XVIe siècle. Les histoires présentent généralement de grands thèmes de réflexion tels les Sept péchés capitaux, la Vertu, le Vice, la Richesse, la Pauvreté, la Connaissance, l'Ignorance ou la Grâce. Chaque comédie s'articule autour d'une figure allégorique appelée l'Homme, représentant les gens du peuple et leur âme. Son antagoniste est le Vice, qui apparaît souvent comme le Diable, capable de ruses et de déguisements. L'Homme est facilement trompé par le Vice, qu'il doit vaincre afin d'atteindre le salut. Les comédies de mœurs sont extrêmement populaires. On peut faire valoir que le roman policier en est un dérivé thématique, l'Homme étant remplacé par le grand détective et le Vice par le méchant meurtrier. Comme dans les comédies, le tueur moderne engendre un état chaotique d'incertitude tandis que le héros détective vient restaurer l'équilibre moral non seulement en arrêtant le meurtrier, mais aussi en expliquant le crime psychologiquement et socialement, dans le sillage des admonestations théologiques médiévales.

Le roman policier a considérablement évolué depuis Poe et Conan Doyle, mais il conserve un sous-texte moralisateur: ou le crime est vaincu ou le chaos moral s'impose. Les magazines de romans feuilletons comme Black Mask, Dime Detective et Detective Fiction Weekly deviennent très populaires entre les années 1920 et le début des années 1940 en présentant des détectives endurcis qui combattent le crime dans les rues des villes modernes, véritables enfers sur terre, où seule une intelligence surhumaine peut rééquilibrer les choses. Dans ses multiples versions, le sous-texte du roman policier vise à rétablir l'ordre moral grâce aux pouvoirs de l'intellect. À moins que l'histoire ne prenne une tournure postmoderne, comme dans certains récits d'Alfred Hitchcock, le détective a des qualités surhumaines symétriques aux compétences sinistres du tueur «diabolique » des temps modernes, qui se cache dans l'obscurité et dont l'identité reste inconnue jusqu'à ce que le détective la dévoile.

L'importance du crime en tant que métaphore de la morale commune est bien visible dans le film Blow-Up (1966) de Michelangelo Antonioni. Un photographe à succès dans la ville de Londres, dont le quotidien s'organise autour de la mode, du jazz, de la drogue, du tabac et du sexe, réalise un jour que sa vie est ennuyeuse, mécanique et dénuée de sens. Tout change après sa rencontre avec une belle jeune femme mystérieuse qui le charme. Il la prend en photo sans cesse. À un moment donné, il remarque quelque chose d'effrayant à l'arrière-plan d'une des photos prises dans un parc. Après avoir étudié l'agrandissement (blow-up, en anglais) de ce cliché, il voit la silhouette d'un cadavre et note des détails qui lui font penser qu'un meurtre a eu lieu. Il se rend sur la scène du crime, mais le corps a disparu. Confus, il commence à le chercher ou du moins à essayer de comprendre la raison de sa disparition. Le tout, en vain. À la fin, il regarde 
un match de tennis (probablement dans un rêve) joué avec des balles imaginaires. La scène du match de tennis s'estompe lentement, au point qu'il ne reste que l'herbe où le corps avait été photographié. Le crime non résolu laisse le protagoniste et le spectateur dans un état de suspension morale. Il s'agit là d'une métaphore de notre incapacité à résoudre les dilemmes de la vie. Quand nous ne parvenons pas à connaître la vérité que dissimule un crime, nous avons tendance à vivre dans une sorte d'angoisse existentielle, livrés que nous sommes à un état d'ambiguïté.

Dupin et Holmes sont des sémioticiens de substitution, qui lisent les signes sur les lieux du crime et réfléchissent à rebours afin de bien comprendre ce que ces signes signifient. C'est influencé par ces deux détectives-sémioticiens fictifs que Peirce considère l'étude des signes comme équivalente à l'étude des indices trouvés sur les lieux d'un crime. Comme Thomas Sebeok et Jean Umiker-Sebeok l'ont fait valoir en 1980, la sémiotique de Peirce est de fait une science de la détection, Peirce une sorte d'expert détective et Holmes, quant à lui, un expert sémioticien.

Bien que Holmes rappelle souvent qu'il ne devine jamais, Sebeok et Umiker-Sebeok ont montré qu'il se livre effectivement à des suppositions: "Tester une hypothèse concernant l'identité d'une personne en recueillant des indices à partir de son apparence physique, de ses schémas discursifs, etc., implique toujours un certain nombre de suppositions, raison pour laquelle Peirce appelle cela "induction par abduction" $»^{7}$. La sémiotique de Peirce et la détection du crime sont, de fait, les deux faces d'un même paradigme intellectuel.

Cette perspective a été brillamment reprise par Umberto Eco dans Le Nom de la rose (1983), le roman où il montre que la détection du crime et l'analyse sémiotique sont ontologiquement indissociables. L'intrigue se déroule au Moyen Âge dans une abbaye où des moines sont assassinés par un tueur en série qui vit parmi eux. Le héros qui enquête sur les crimes est un franciscain érudit, Guillaume de Baskerville - un nom qui fait allusion de façon évidente à un grand classique de Sherlock Holmes, Le Chien des Baskerville (1901-1902), et à son décor sombre et inquiétant. Le moine résout les crimes de la même manière et dans le même style que Holmes, interprétant les signes laissés par le tueur sur chaque scène de crime par le biais d'une brillante démonstration de raisonnement abductif, rassemblant les indices de manière à raconter l'histoire du crime et à dévoiler les intentions du tueur. En même temps, Eco utilise cette situation comme une métaphore du changement dans la mentalité médiévale, qui s'éloigne d'une vision du monde strictement religieuse pour s'orienter vers une vision de plus en plus laïque, qui donnera le jour progressivement à l'humanisme et à la Renaissance.

Dans Une étude en rouge, Holmes explique qu'il parvient à la solution du crime grâce à la collecte intuitive d'indices, à un enchaînement de pensées qu'il arrive à canaliser dans un raisonnement coordonné. Lorsque Watson lui demande ce qu'il entend par là, dans une histoire subséquente, Le signe des quatre (1890), Holmes répond: «Ah, c'est de la chance. Je ne pouvais dire que ce qui me paraissait le plus probable. Je ne m'attendais pas du tout à être aussi exact ${ }^{8}$. Ce qui est bel et bien une description de l'abduction (involontaire, bien sûr). De nos jours, même si nous disposons de puissants outils médico-légaux, comme l'analyse de l'ADN, la solution d'une affaire criminelle commence toujours par ce genre de collecte intuitive d'indices. Il est intéressant de noter que Conan Doyle, en 1877, était étudiant en médecine à l'Université d'Édimbourg, où l'un de ses professeurs, le Docteur Joseph Bell, était un pionnier de la médecine légale. Bell a appris à Conan Doyle à envisager l'enquête criminelle comme un mélange 
de science et d'art de l'inférence. Doyle est devenu l'assistant de Bell et a pu observer de près les compétences de son mentor en matière de détection. Bell faisait souvent des remarques sur l'importance de la démarche et des inflexions linguistiques des étrangers comme autant d'indices potentiels pour établir l'identité d'un criminel. Aussi incroyable que cela puisse paraître, les intuitions de Bell étaient généralement justes. Conan Doyle en a été impressionné au point de prendre son mentor pour modèle lors de la création de son détective fictif. Comme le fera Holmes avec Watson, il semblerait que Bell ait souvent dit à son disciple que tout cela était « élémentaire ».

Les histoires de Sherlock Holmes sont tout à la fois des essais de médecine légale, de criminologie, de méthode sémiotique et de psychologie de l'esprit humain ${ }^{9}$; Conan Doyle a certainement compris qu'il y a une dimension morale inhérente à la criminalité et que l'on peut saisir la nature même de la morale en examinant les crimes et leurs auteurs. Les romans de Sherlock Holmes sont devenus des piliers de la culture littéraire et médico-légale moderne. On pourrait dire, en fait, que Holmes a été le premier sémioticien judiciaire puisqu'il a utilisé la détection et l'identification des signes pour élucider des crimes.

En somme, les auteurs de romans policiers ont toujours été très conscients du pouvoir des symboles dans les récits de détection. Crime et châtiment de Dostoïevski ou les histoires d'horreur de Poe sont en réalité des narrations fondées sur la sémiotique judiciaire car ils montrent les forces non rationnelles et, dans certains cas, irrationnelles, qui caractérisent le comportement criminel. Songeons que certains tueurs en série pratiquent la nécrophilie afin de revivre leurs crimes par procuration, grâce à la présence symbolique du corps qui réactive les échos et l'excitation associés au crime et à ses fonctions antirépressives. Autant d'instants de revitalisation qui répondent au besoin du tueur de réifier sa subjectivité. C'est là que la conscience de soi du tueur fait surface en tant que locus de contrôle. Les corps sont des trophées qui représentent des signes de la conscience de soi; les lieux du crime deviennent des endroits où l'auto-construction peut se déployer. L'un des postulats fondamentaux de la sémiotique judiciaire est qu'il existe une continuité entre crimes fictifs et crimes réels ${ }^{10}$ - ils sont le reflet l'un de l'autre et s'influencent l'un l'autre. Pour un cas d'espèce, que l'on pense au phénomène des tueurs imitateurs ${ }^{11}$. La plupart de ceux ou celles qui imitent les crimes médiatisés ont un casier, de graves problèmes de santé mentale ou des antécédents de violence, ce qui laisse supposer que l'effet des médias est indirect. Mais il y a des cas qui montrent que des individus veulent simplement paraître sous les feux de la rampe en tant que tueurs en série. Le crime donne à certaines personnes un sentiment de puissance et une identité qu'elles désirent et qu'elles ne peuvent sans doute pas atteindre autrement.

\section{Sémiotique judiciaire}

L'expression sémiotique judiciaire a été utilisée en 2007 par William Buckland ${ }^{12}$ pour désigner les styles d'écriture et les caractéristiques qui peuvent servir à identifier un auteur (longueur des phrases, modules lexicaux, fréquence des mots, etc.). La définition que nous avons proposée en 2013 de la sémiotique judiciaire comme branche à la fois de la sémiotique, de la criminologie et de la médecine légale, si elle permet de souligner à quel point Peirce admirait les récits de Poe et Conan Doyle, a surtout pour but 
d'appliquer la sémiotique à l'étude tant de la détection criminelle que de la question plus générale du rapport que le crime entretient avec le sens, la culture et l'histoire.

De même que les significations de la sexualité et de la maladie mentale sont liées à l'expérience, au savoir et à l'histoire ${ }^{13}$, ainsi les significations d'un crime ne peuvent être déterminées dans l'absolu, mais uniquement en tenant compte des spécificités d'un contexte. À quelques exceptions près, tuer en temps de guerre n'est pas un meurtre; mais si le même soldat tuait la même personne en dehors de la guerre, cela serait considéré comme un homicide. Le fait de qualifier tel ou tel acte comme un crime ou un meurtre, exige une interprétation qui tienne compte de tel ou tel environnement culturel.

Plus les médecins légistes et les criminologues prendront conscience de la façon dont les signes manifestent le fonctionnement de l'esprit criminel, plus ils seront en mesure de comprendre le crime, comme le savaient certainement les premiers auteurs de romans policiers et comme Peirce l'a souligné à son tour. Deux manières directes d'exploiter la sémiotique sont possibles ${ }^{14}$. La première peut être appelée sémiotique judiciaire de premier niveau (SJ1). L'objectif de la SJ1 est d'appliquer la théorie des signes et les pratiques d'analyse des signes aux preuves recueillies par les experts médicolégaux. Elle peut surtout aider les enquêteurs à comprendre comment les expressions faciales, les gestes, le langage et d'autres systèmes de signes peuvent être utilisés pour déceler la tromperie et identifier les auteurs d'actes criminels (Bell, le mentor de Conan Doyle, l'avait bien compris). La deuxième manière doit permettre d'évaluer le lien entre la criminalité, les représentations fictives (et médiatiques) de la criminalité et les définitions et perceptions culturelles de la criminalité. L'étude de ce lien peut être qualifiée de sémiotique judiciaire de second niveau (SJ2).

La SJ2 a commencé à intéresser bon nombre de criminologues, bien qu'elle ne soit pas toujours reconnue comme telle. Il existe une importante revue, Crime, Media, Culture, qui publie des recherches sur la relation entre la criminalité, la justice pénale, les médias et les tendances culturelles. L'un des phénomènes les plus remarquables qu'étudient les criminologues s'appelle effet multiplicateur du crime. Son nom fait référence à l'impact que, par le biais de l'exposition médiatique, des faits criminels réels peuvent avoir sur les perceptions et les comportements. La poursuite d'O.J. Simpson, télévisée en 1994 en temps réel, suivie du procès de O.J. Simpson, également transmis en direct, sont emblématiques de cette dynamique. Simpson étant lui-même une icône des médias, l'événement est devenu un spectacle qui a largement influencé l'opinion quant à sa culpabilité ou son innocence dans le meurtre de son ex-femme Nicole Simpson et de son ami Ronald Goldman. Par ailleurs, étant donné le sous-texte racialisé de l'affaire, le procès s'est transformé en un procès où la justice sociale était un enjeu. L'affaire Simpson a démontré que les procès médiatisés sont des représentations théâtrales qui influencent l'issue des procédures, en plus d'être des comédies de mœurs semblables aux classiques du genre ${ }^{15}$.

A l'Université de Toronto, l'analyse sémiotico-judiciaire a été appliquée à une affaire de meurtre ${ }^{16}$, l'affaire non résolue de David Buller, un professeur assassiné dans son bureau, sur le campus, le 18 janvier 2001. Buller était un artiste visuel. Par conséquent, le point de départ pour résoudre l'affaire était son œuvre, qui permettait au sémioticien de donner du sens à sa vie et de vérifier si des signes présents dans ses travaux pouvaient se référer à une période, à une personne ou à un événement qui 
seraient éclairants pour l'enquête. L'affaire avait fait l'objet d'une enquête mais avait été classée et avait vite disparu des unes des journaux et de la mémoire du campus.

Treize ans plus tard, j'ai été approché par une ancienne étudiante en sémiotique, Renée Willmon, qui estimait que je serais en mesure de faire la lumière sur cette affaire, étant donné que Buller avait laissé des tableaux susceptibles de conduire à la solution. Extrêmement sceptique et néanmoins intrigué, je ne savais rien de l'enquête policière et je n'avais aucun souvenir des détails de l'affaire. Essentiellement, on m'a demandé de donner une interprétation sémiotique des peintures de Buller pour vérifier si elles recelaient des indices sur le meurtre. Je suis allé à une exposition de Buller en juillet 2013 sans aucune information sur son passé ou sa vie privée, ni aucun détail sur la scène du crime. De toute évidence, les premières œuvres de Buller imitaient Andy Warhol et elles révélaient également son orientation sexuelle. Mais ses deux derniers tableaux étaient particulièrement significatifs. L'un semblait représenter ce que les experts médico-légaux appellent une scène d'éclaboussures de sang, avec un individu peint à l'arrière-plan, comme s'il jaillissait de l'esprit du peintre. Je ne savais pas à l'époque comment Buller était mort, mais j'ai demandé s'il avait été poignardé plusieurs fois et s'il y avait beaucoup de sang sur les lieux du crime. On a répondu par l'affirmative à mes deux questions. J'en ai conclu que le tableau préfigurait la mort de l'artiste. Il y avait aussi un côté clair-obscur dans cette œuvre où se juxtaposaient la peur profonde du peintre et ce monde plus lumineux qui caractérisait ses œuvres antérieures. La clarté a vite disparu de la vie de Buller. L'autre tableau était une sorte de mise en exergue définitive du sentiment de peur et de danger qu'il ressentait. La silhouette d'un homme n'a cessé d'apparaitre dans l'œuvre peint de Buller, mais dans son dernier tableau elle a disparu dans l'obscurité. J'ai supposé qu'il s'agissait d'un amant éconduit dont Buller craignait qu'il puisse le tuer. J'ai présenté cette conclusion aux conservateurs du musée, qui étaient des amis du professeur assassiné, et ils m'ont informé que la police avait bien identifié cet homme comme l'auteur probable du crime, mais n'avait pas suffisamment de preuves pour l'arrêter. Ce qui leur a semblé particulièrement intéressant, c'est que j'étais parvenu à la même conclusion en utilisant la méthode sémiotique.

Cette affaire a montré à quel point la sémiotique peut être utile dans le cadre d'une enquête et pour expliquer un crime. Dans les tableaux de David Buller, il y avait des indices clairs de son état d'esprit : il avait senti que sa fin était imminente. De toute évidence, les signes que Buller a laissés dans ses deux dernières œuvres sont susceptibles d'être utilisés rétroactivement par les enquêteurs.

Il en va de même lorsqu'il s'agit de textes écrits ou oraux. J'ai pu vérifier cette hypothèse avec Michael Arntfield, criminologue et ancien policier : nous avons analysé les écrits et les déclarations de vrais meurtriers et ceux d'auteurs de romans policiers, les avons comparés pour voir si l'analyse sémiotique permettait d'obtenir des informations pertinentes sur l'esprit des criminels. Le résultat est une étude où nous avons montré comment les textes écrits donnent effectivement un aperçu de l'état d'esprit d'un criminel ${ }^{17}$. L'analyse de notes manuscrites, de déclarations et de souvenirs de meurtriers nous a permis de dresser un profil psycho-graphique global de chaque individu dans son contexte. Nous sommes même parvenus à identifier un tueur en série inconnu à l'époque. Les détails le concernant ont été rendus publics un an plus tard. 


\section{Sémiotique et culture mafieuse}

Le crime organisé est l'autre secteur d'enquête auquel j'ai appliqué la sémiotique judiciaire, en travaillant avec l'historien de la mafia Antonio Nicaso ${ }^{18}$. L'analyse des symboles et des rituels utilisés dans la culture mafieuse - ces symboles et ces rituels qui confèrent un sens d'autorité métaphysique à l'organisation criminelle- a été particulièrement utile à notre approche globale.

Rejoindre la mafia, c'est comme rejoindre une sorte d'ordre religieux, avec ses serments, ses rites, ses pratiques symboliques, ses règles de comportement, ses châtiments, etc. L'omertà, à savoir le code d'honneur et de la virilité mafieuse, est en même temps un code religieux sur mesure et un code pseudo-chevaleresque. Ce lien avec la religion et la chevalerie paysanne (cavalleria rusticana) permet à la mafia de s'insérer sans accrocs dans le substrat culturel du sud de l'Italie.

Nous avons découvert que ce sont les rituels et les symboles qui donnent à l'appartenance à la mafia sa stabilité et sa signification. Sans eux, l'organisation criminelle aurait plus de difficulté à maintenir les liens affectifs essentiels qui unissent ses membres. Les rituels marquent en effet les événements cruciaux et récurrents de la vie de l'individu et d'une communauté : la naissance, le mariage, la mort. L'une des cérémonies les plus importantes pour les groupes criminels est celle qui sert à initier au clan de nouveaux membres en les transformant en affiliés. L'initié sait très bien que pour devenir un homme nouveau, un affilié donc, il devra symboliquement rejeter son passé et sa vraie famille, en faisant allégeance exclusive au clan, qui devient alors sa seule famille.

Comme chacun sait, on ne saisit pas la réalité directement, mais par le biais de signes (mots, symboles) qui permettent de l'évaluer. Nos signes et systèmes de signes sont fondamentalement fictifs, parce qu'ils ne racontent pas la véritable histoire de l'existence, mais seulement une version sélective et interprétative de celle-ci. Les organisations criminelles ont certainement compris ce principe de base de la sémiotique et ont manipulé les faits en utilisant la fiction afin de produire leur propre mystique $^{19}$. En fait, une grande partie de la mystique de la mafia telle qu'on la voit à la fois dans les films et dans la vie réelle, est fondée sur des symboles associés au mysticisme rituel (symboles empruntés aux ordres religieux, aux sociétés secrètes et à d'autres groupes), à la mystification (fragments de langage isolés) et aux codes de présentation de soi. Il y a dans les rituels de la mafia, une mise en scène, une dramatisation, même si les cérémonies proprement dites sont dénuées de charme et plutôt ennuyeuses, vues de l'extérieur.

Les groupes criminels sont ce qu'Emile Durkheim a appelé des sociétés segmentaires ${ }^{20}$, c'est-à-dire des sociétés organisées par composantes dont la survie dépend d'une solidarité mécanique, obtenue par la reproduction rituelle de formes culturelles existantes. Le rituel est un premier pas à accomplir pour accéder à la reproduction et obtenir ainsi le consensus des nouvelles recrues. Le rituel recrée ainsi l'identité de tout nouveau membre, la façonnant selon les attentes du groupe. L'objectif étant de donner aux nouvelles recrues un nouveau système de croyances et un nouveau rôle dans une nouvelle famille, mais aussi des consignes précises quant à la manière d'interagir avec autrui. Comme l'a remarqué John Dickie, «s'agissant de la mafia, le rituel d'initiation renforce spécialement les mythes relatifs à l'ancienneté de l'organisation. En réalité, celle-ci est aussi moderne que tout ce qui concerne la mafia $»^{21}$. La première description 
d'un rituel d'allégeance se trouve dans un rapport de police rédigé en 1876 à Palerme, qui concerne le clan mafieux dirigé par Antonio Giammona, l'un des premiers chefs mafieux bien connus de l'Italie post-unitaire. À peu près à la même époque, la mafia décide de propager la mythologie de ses origines, qui remonteraient à de nobles chevaliers d'antan - un fabuleux récit qui intègre la bande criminelle dans un substrat culturel mythique.

Les groupes criminels racontent invariablement des histoires faisant remonter leurs origines à un mythe ou à une fable, afin de véhiculer une impression de vérité historique et de légitimité. En réalité, la criminalité en Sicile est la conséquence d'un système féodal inscrit dans le temps long, socialement inégalitaire. Les nobles, oisifs et absents, avaient besoin d'hommes solides pour la gestion de leurs domaines, afin de préserver localement leur pouvoir et leur influence sans forcément se montrer très respectueux des lois. Les aristocrates engageaient des bandits comme gardes personnels (guardiani). La corruption était endémique. Beaucoup de juges achetaient leurs postes; les greffiers étaient peu ou pas payés. N'ayant aucune confiance en la justice, la population locale a cherché d'autres moyens de se protéger. Le banditisme s'est très tôt imposé comme un racket de protection qui, au cours du XIXe siècle, s'est répandu dans toute la société sicilienne. Le ralliement des bandits pendant la rébellion de 1848 contre les Bourbon-Siciles relevait de l'opportunisme: la mafia a gagné en légitimité et en pouvoir puisque les combattants pour l'Unité italienne considéraient l'organisation leur alliée. C'est «la méfiance sicilienne traditionnelle envers les institutions de l'État qui a créé les conditions dans lesquelles la mafia a pu se développer $»^{22}$.

Que l'on se réfère au cas des trois mafias du sud de l'Italie (la Mafia sicilienne, la 'Ndrangheta calabraise et la Camorra napolitaine) : un mythe fondateur a été construit de toutes pièces pour les unir, la Garduña, un groupe créé dans les prisons d'Espagne et qui serait devenu une société criminelle clandestine dont la principale activité était le meurtre sur commande. Une légende calabraise raconte que trois frères de la Garduña ont quitté l'Espagne après avoir tué un noble qui avait agressé leur sœur. Après avoir fait naufrage sur l'île de Favignana, l'un d'entre eux s'est rendu à Naples, où il a fondé la Camorra, un deuxième en Calabre, où il a fondé la 'Ndrangheta, et un troisième en Sicile, où il a fondé la Mafia. Cette légende est un récit allégorique qui permet d'établir un lien entre les bandes criminelles et la culture de la piraterie de l'Empire espagnol. L'histoire a montré que la 'Ndrangheta, tout comme la Mafia, est apparue lorsque les intérêts de l'organisation criminelle coïncidaient avec ceux des grands propriétaires terriens et des notables pour contrôler les élections afin de maintenir le statu quo, mais le criminologue qui ne tiendrait pas compte des aspects mythologiques que nous venons d'évoquer délaisserait une des fonctions sémiotiques essentielles des récits historiques : celle qui permet de cerner la réalité culturelle.

La stratégie mythologique est imbriquée avec l'intertextualité. C'est pourquoi des citations bibliques jalonnent tous les documents des bandes criminelles réquisitionnés par la police. Ces bribes de texte confèrent une aura religieuse aux activités criminelles. La Sacra Corona Unita, organisation basée dans la région des Pouilles, est allée jusqu'à prendre le nom d'un artefact religieux parmi les plus significatifs, la sacra corona (le chapelet sacré), dans le but de souder les membres du groupe comme dans une secte. Lors de perquisitions dans les maisons des mafieux, la police a découvert partout divers icônes et objets prouvant que les clans criminels utilisent systématiquement le lien 
avec la religiosité. La Mafia est considérée comme un gang honorable en partie en raison de sa fidélité aux pratiques traditionnelles et de son aversion pour les valeurs matérialistes du monde moderne, qu'elle considère vaines et contraires aux traditions et à l'histoire de l'Italie authentique.

Comme nous l'avons dit, les rituels essentiels pour tout groupe criminel sont ceux qui amènent un nouveau membre dans le giron de l'organisation. Or, dans la Mafia, afin d'être éligible à l'initiation, le membre potentiel doit souvent participer à un meurtre cela s'appelle farsi le ossa (littéralement : se faire les os). Le candidat attendra ensuite qu'on lui demande d'assister à une réunion spéciale du clan, avec des membres assis autour d'une table située à un endroit supposément sacré. Après avoir répondu aux questions du clan, l'initié intègre la véritable procédure d'entrée dans le rôle de chevalier mafieux. Le rituel consiste justement à tenir entre ses mains une image pieuse en flammes et à réciter un serment qui engage au secret et à l'obéissance. Le chef de clan prend alors un couteau et incise le doigt de la gâchette, l'index de l'impétrant. Le sang qui coule du doigt symbolise à la fois sa mort et celle de ceux qu'il pourrait devoir tuer. La mort de l'initié est envisagée au sens métaphorique (il abandonne sa vie antérieure pour renaitre dans une nouvelle vie) et littéral, puisque pour tout manquement au serment il sera condamné à périr. La partie du rite marquée par les flammes renvoie également à la mort métaphorique. Le feu lie l'initié au clan pour la vie :

L'initiation consistait en un serment de sang et en un serment d'obéissance.

L'aspirant devait être présenté à l'initiation par au moins trois "hommes d'honneur" de la famille. Du sang était prélevé du doigt de l'initié et versé sur l'image d'un saint, que l'on brûlait et l'on passait de main en main pendant que l'initié jurait de respecter le code de Cosa Nostra, auquel il était uni pour la vie. Sa cosca était sa nouvelle famille et il ne pourrait changer d'obédience ${ }^{23}$.

Dans certains clans, le feu est utilisé pour brûler des parties du corps afin de prouver la loyauté par le courage. Le feu représente la purification et l'acquisition de la sagesse. Ainsi purifié, l'initié est digne d'être appelé membre du clan. Mais le feu est aussi emblématique de la prise de risques, car on attend du mafieux, symboliquement et concrètement, qu'il fasse preuve de courage et de fidélité en supportant les flammes.

En plus des rites d'initiation et de passage, il existe des rites (explicites ou implicites) qui régissent pratiquement tous les aspects de la vie clanique. Pour commémorer la mort d'un mafieux, par exemple, les femmes doivent s'habiller en noir et montrer leur chagrin aux funérailles en priant à haute voix et en pleurant sur le cercueil. Pendant ce temps-là, le capo ou un autre membre de rang élevé, va vers la mère ou l'épouse du défunt pour la serrer dans ses bras et la consoler. On s'attend cependant à ce qu'elle rejette son geste, en lui frappant la poitrine avec ses poings. Le mafieux doit néanmoins insister à la consoler; elle, de son côté, doit enfin lui permettre de l'embrasser jusqu'à ce qu'elle cesse de sangloter et montre sa résignation. La femme signale ainsi qu'elle accepte le rôle de la mafia dans sa vie et reconnaît être soumise aux principes de l'omertà. Comme l'explique Lunde :

Le vrai mafieux s'occupe de sa famille criminelle comme un père, servant souvent de parrain aux enfants de ses subalternes, assistant à leurs mariages et à leurs funérailles, et organisant des banquets fréquents, presque rituels, où la disposition des sièges correspond au statut que chacun occupe dans la famille ${ }^{24}$.

La pertinence de la sémiotique judiciaire dans l'étude de la culture mafieuse réside dans sa capacité à identifier les pratiques symboliques qui deviennent intrinsèques à 
l'identité et à la cohésion du groupe. Comme nous l'avons vu plus haut, les rituels sans symboles n'auraient littéralement aucun sens. Ils donnent une dimension mémorable aux événements et n'exigent aucune science ou théorie explicative. Les symboles utilisés par la mafia, tels le sang, le feu, les mains, sont plutôt courants et renvoient à une sorte d'instinct symbolique. Le symbolisme des liens du sang dans la culture mafieuse est décrit comme suit par Lunde :

Dans un monde où le pouvoir était arbitraire, la sécurité passait par la famille. Plus le groupe familial était grand et étendu, plus l'individu était protégé, surtout dans un système social de vendettas, où l'honneur exigeait qu'aucune offense ne soit laissée impunie. Une armée de frères et de cousins était la meilleure protection dans un monde injuste. Rien n'est plus important pour un Sicilien que les liens du sang $^{25}$.

D'après Reynolds :

Une grande partie du glamour et de l'intrigue que les étrangers associent à Cosa Nostra provient de l'omertà, le code d'honneur scellé dans une cérémonie secrète $d^{\prime}$ initiation qui confère à ses membres le caractère sacré du code ${ }^{26}$.

Ce n'est pas un hasard si le symbole de la mafia calabraise est d'origine biblique, à savoir l'arbre de la connaissance, composé d'un tronc (le chef de clan) exerçant le pouvoir sur ses appendices, les branches et les feuilles.

\section{Penser comme Sherlock Holmes}

Les modes d'analyse utilisés par Sherlock Holmes pour résoudre les crimes ne sont pas très différents de ceux utilisés aujourd'hui en médecine légale. Les deux sont inhérents au décodage logique et cohérent des signes de la criminalité. Dans Le Chien des Baskerville, le visage terrifié et déformé de la victime, Sir Charles Baskerville, est censé représenter la terreur ressentie en affrontant le chien fantôme dont parle la légende familiale. La lanterne est utilisée pour observer les empreintes de pas et leur signification. Holmes infère que les empreintes de pas de l'homme montrent qu'il courait, terrifié par un gros chien, qui à son tour a laissé des traces. Une scène qui semble correspondre à la légende selon laquelle un chien tue tous les héritiers Baskerville.

À un moment donné, on trouve une lettre qui s'avère être un stratagème élaboré pour effrayer Baskerville, la victime désignée du chien fantôme. On saura par la suite que cette lettre a en fait été écrite par la femme du suspect principal, mais de manière à cacher qu'elle en est l'auteur. On découvre qu'elle ne voulait pas que son mari persiste dans son projet et qu'elle l'a donc écrite pour essayer de lui faire peur. Pour Holmes, l'arôme de White Jasmin qui se dégage de la lettre renvoie à la femme, qui en effet porte ce parfum. Malgré l'insistance de son mari, elle refuse de seconder ses projets criminels par conscience morale. L'échantillon de terre prélevé dans le van à chevaux vers la fin de l'histoire a un lien avec le suspect, parce que ce dernier a creusé des fossés dans la bruyère. La terre retrouvée dans le van, semblable à la terre de la bruyère, a été transportée de la bruyère jusqu'aux bottes du suspect puis au van.

Le raisonnement utilisé par Holmes est vraiment impressionnant. Les traits déformés du visage sont d'abord considérés comme les symptômes d'une crise cardiaque, mais ensuite, Holmes a informé le médecin légiste que c'est la réaction à un chien de chasse monstrueux qui a provoqué la crise cardiaque, d'où l'expression terrifiée du visage. 
La lettre anonyme ne pouvait être écrite que par une personne cultivée, puisqu'elle a été composée avec des découpages de journal, tandis que l'écriture précaire qui figurait sur l'enveloppe (l'adresse était manuscrite) ne correspondait pas au style d'une personne capable de lire un journal. Holmes a déclaré qu'il s'agissait « évidemment » de la tentative d'une personne éduquée de faire croire qu'une personne sans éducation avait concocté ce courrier. Du papier se dégageait une senteur qui, comme nous l'avons vu, s'est avérée être du White Jasmin, l'essence du parfum que porte la femme du suspect.

L'importance de Sherlock Holmes pour le développement de la criminologie moderne a été bien soulignée à la fois par Maria Konnikova et par James $0^{\prime} B^{3}{ }^{2}{ }^{27}$, mais aucun des deux n'a fait référence à Peirce ou à la sémiotique. Or, si on se réfère à Peirce, on comprend que ce qui nous attire vraiment, c'est la capacité de raisonnement de Holmes. Konnikova remarque en effet que celle-ci implique l'observation en plus de la détection. Konnikova distingue d'ailleurs le "système Watson ", à savoir la tendance naturelle à croire ce que l'on entend et ce que l'on voit, du « système Holmes ", qui fait intervenir le questionnement, l'observation et la présence d'esprit. Il s'agit d'une sorte de processus dialectique qui génère constamment des hypothèses et des inférences jusqu'à ce que celles-ci soient réduites à une seule conclusion, la seule qui puisse être liée à un crime donné. Et c'est précisément ainsi que fonctionne l'abduction.

Les enquêtes sur les lieux d'un crime sont efficaces non seulement parce qu'elles font appel à une série de méthodes et de procédures scientifiques, mais également ou surtout parce qu'elles constituent une forme d'analyse sémiotique à la Sherlock Holmes. Tout indice a une pléthore de significations possibles, qui doivent être examinées dans le contexte de la scène du crime, à la lumière des connaissances culturelles et historiques pertinentes et des informations scientifiques disponibles, afin d'être correctement interprétées. Même si les premières hypothèses s'avèrent fausses, le positionnement de certains signes par rapport à d'autres signes présents sur les lieux peut conduire les enquêteurs vers un système logiquement connecté, qui peut être modifié ou même rejeté. Comme Holmes, les experts en sciences judiciaires résolvent des crimes déconcertants grâce à l'observation intelligente et à l'abduction.

\section{Conclusion}

Aujourd'hui, la police et les criminologues sont conscients du fait que pour comprendre l'étiologie du comportement criminel et des groupes du crime organisé, il faut prendre au sérieux les rituels, les symboles et les différentes pratiques fondés sur les signes. Certains services de police commencent à utiliser l'outil sémiotique dans les enquêtes criminelles. Songeons notamment au Center for Homicide Research de la NouvelleOrléans, qui a adopté des techniques sémiotiques pour enquêter sur les scènes de crime et pour décoder les symboles utilisés par les criminels et les gangs. Les tribunaux commencent également à utiliser la preuve sémiotique dans les procédures pénales. Dans la ville d'Edmonton, au Canada, par exemple, le tribunal vérifie si l'accusé utilise ou non un nom, un mot, un symbole ou une autre forme d'expression qui se réfère ou est associé à une organisation criminelle, afin de déterminer si l'accusé en fait partie.

Un autre événement marquant a été, en 2015 , le procès d'un Californien accusé d'avoir tenu un comptoir de marché noir en ligne. Au cours du procès, qui a eu lieu à New York, l'avocat de l'accusé a soulevé une objection suggérant qu'un élément de preuve 
essentiel avait été omis par l'accusation, à savoir un smiley. L'objection a été soulevée après la lecture par le procureur d'un message Internet écrit par l'accusé, sans mentionner l'émoji au visage heureux qui l'accompagnait. L'avocat prétendait que l'utilisation de cet émoji montrait que son client n'était pas sérieux, mais facétieux, et qu'il n'avait donc aucune intention criminelle. Le juge a demandé au jury de prendre en compte ce symbole. Autrement dit, un émoji a été accepté dans un tribunal en guise de preuve des intentions d'un individu, à savoir comme révélateur de son état d'esprit et comme support de ses déclarations ou de ses aveux. Il s'agit là d'une application indirecte de la sémiotique judiciaire, à partir d'un symbole visuel.

La même année, la police de Pittsburgh a également présenté trois émojis utilisés dans un texto, comme preuve dans un procès pour double homicide. L'accusation a soutenu que les émojis serviraient à démontrer que l'expéditeur, qui avait été lui-même blessé lors d'un vol qualifié, était responsable du coup de feu mortel. L'expéditeur a passé cinq jours dans le coma avant de reprendre connaissance. L'accusation a affirmé que les émojis envoyés avant la fusillade montraient qu'il avait l'intention de participer au vol qui a mené aux meurtres. Les émojis représentaient un homme qui courait, une explosion et une arme à feu. Cette affaire montre de toute évidence que la sémiotique judiciaire est implicitement présente aux esprits et qu'il reste juste à en faire un usage explicite.

Cet article, on l'aura compris, plaide en faveur d'un emploi concret et assumé de la sémiotique judiciaire. La criminalité est en train de se transformer en phénomène planétaire, dans le village global qui est le nôtre, ce qui rend les outils de la sémiotique encore plus pertinents pour la combattre. Tout, des attaques terroristes à la fraude ordinaire, est annoncé, planifié et présenté via des sites Internet. Y a-t-il une dynamique sémiotique qui unit la criminalité on-line et la criminalité off-line ? Ou bien la première est-elle devenue plus puissante ? La sémiotique judiciaire peut certainement enquêter sur la question, en examinant cette nouvelle réalité complexe à laquelle sont confrontés la police et les légistes.

Internet a fait du mensonge, de la dissimulation et de la manipulation des modes communs d'interaction. Elle a permis au menteur pathologique ou compulsif de faire entendre sa voix et de la diffuser largement. La société médiévale avait des monstres mythiques et des démons imaginaires pour expliquer le mal; nous avons de vrais tueurs. Comme Mikhaïl Bakhtine l'a souvent dit, les monstres mythiques sont nécessaires pour restaurer notre croyance dans le sacré moyennant un combat acharné contre ses adversaires ${ }^{28}$. Par conséquent, la relation entre le crime et les systèmes de signes, entre le crime et l'histoire gagne à être affrontée par le biais de l'analyse sémiotique.

\section{BIBLIOGRAPHY}

Arntfield, Michael A. (2016), Gothic Forensics : Criminal Investigative Procedure in Victorian Horror and Mystery, New York, Palgrave-Macmillan. 
Arntfield, Michael A. et Danesi, Marcel (2016) (Eds.), The Criminal Humanities, New York, Peter Lang.

Arntfield, Michael A. et Danesi, Marcel (2017), Murder in Plain English : From Manifestos to Memes Looking at Murder through the Words of Killers, New York, Prometheus.

Bakhtine, Mikhaïl (1978), Esthétique et théorie du roman, Paris, Gallimard.

Buckland, William (2007), « Forensic semiotics », Semiotic Review of Books, 10 (3), pp. 9-16.

Coleman, Loren (2004), The Copycat Effect : How the Media and Popular Culture Trigger the Mayhem in Tomorrow's Headlines, New York, Simon and Schuster.

Cotterill, Janet (2000), Language and Power in Court: A Linguistic Analysis, New York, Palgrave. Danesi, Marcel (2013), Signs of Crime : An Introduction to Forensic Semiotics. Berlin, Mouton de Gruyter.

Danesi, Marcel (2014), « Behind the Scenes at TCAK : Semiotics in the Service of Criminal Investigations », https://reneewillmon.wordpress.com/tag/research-in-forensic-semiotics/ Dickie, John (2007), Cosa Nostra. L'histoire de la mafia sicilienne de 1860 à nos jours, Paris, BuchetChastel.

Durkheim, Emile (1912), The Elementary Forms of Religious Life, New York, Collier.

Eco, Umberto et Sebeok, Thomas A. (Eds.) (1983), Dupin, Holmes, Peirce: The Sign of Three. Bloomington, Indiana University Press.

Foucault, Michel (1969), L'Archéologie du savoir, Paris, Gallimard

Gambetta, Diego (1993), The Sicilian Mafia: The business of private protection, Cambridge, Harvard University Press.

King, B. H. et Ford, C. V. (1988), « Pseudologia fantastica », Acta Scandinavica, 77, 1-6.

Konnikova, Maria (2013), How to think like Sherlock Holmes. New York, Viking.

Lombroso, Cesare (1876), L'uomo delinquente, in rapporto all'antropologia, alla giurisprudenza ed alla disciplina carceraria, Milano, Hoepli.

Lunde, Paul (2004), Organized Crime: An Inside Guide to the World's Most Successful Industry, London, Dorling Kindersley.

McNab, Chris (2010), Serial Killer Timelines, Berkeley, Ulysses Press.

Nicaso, Antonio et Danesi, Marcel (2013), Made Men : Mafia Culture and the Power of Symbols, Rituals, and Myth, Lanham, Rowman \& Littlefield.

O'Brien, James (2013), The Scientific Sherlock Holmes : Cracking the Case with Science and Forensics, Oxford, Oxford University Press.

Peirce, Charles S. (1958), Collected Papers of Charles Sanders Peirce, Vols. 1-8, edited by C. Hartshorne and P. Weiss, Cambridge (MA), Harvard University Press.

Reynolds, John L. (2006), Shadow People : Inside History's Most Notorious Secret Societies, Toronto, Key Porter Books.

Sebeok, Thomas A. et Umiker-Sebeok, Jean (1980), You Know My Method : A Huxtaposition of Charles S. Peirce and Sherlock Holmes, Bloomington, Gaslight Publications.

Smith, Dwight C., Jr. (1975), The Mafia Mystique, Chicago, University of Chicago Press. 
Thomas, Ronald R. (1999), Detective Fiction and the Tise of Forensic Science. Cambridge, Cambridge University Press.

\section{NOTES}

1. Cf. Arntfield 2016.

2. Cf. Thomas 1999.

3. Cf. Danesi 2013 et Arntfield 2016.

4. Cf. Peirce 1931.

5. Cf. Danesi 2013 et Arntfield et Danesi 2016.

6. Cf. Eco et Sebeok 1983.

7. Sebeok et Umiker-Sebeok 1980, p. 63. Sauf précision contraire, toutes les citations en langue étrangère sont livrées en version française par la traductrice de ce texte.

8. La citation est tirée de la version française du roman disponible en ligne à l'adresse http:// www.crdp-strasbourg.fr/je_lis_libre/livres/CononDoyle_LeSigneDesQuatre.pdf (p. 12).

9. Cf. Konnikova 2013 et O'Brien 2013.

10. Cf. Arntfield et Danesi 2017.

11. Cf. Coleman 2004.

12. Cf. Buckland 2007.

13. Cf. Foucault 1969.

14. Cf. Danesi 2013.

15. Cf. Cotterill 2002.

16. Cf. Danesi 2014.

17. Cf. Arntfield et Danesi 2017.

18. Cf. Nicaso et Danesi 2013.

19. Cf. Smith 1975.

20. Cf. Durkheim 1912.

21. Cit. par Lunde 2004, p. 37.

22. Id., p. 55.

23. Id., p. 68 .

24. Id., p. 55.

25. Ibid.

26. Reynolds 2006, p. 192.

27. Cf. Konnikova 2013 et O'Brien 2013.

28. Cf. Bakhtine 1978.

\section{ABSTRACTS}

The science of crime, or forensic science, crystallized at around the same time as gothic and detective literature in the latter part of the nineteenth century. A large portion of the early crime-detection techniques of the era were derived from, or modeled on, those employed by fictional detectives such as C. Auguste Dupin and Sherlock Holmes. Vice versa, new forensic techniques were incorporated by fiction writers so as to make their stories more realistic, and in 
line with the emerging cultural scientism of the era. The connection between forensic science and crime fiction continues to this day. This merger can be called forensic semiotics, which aims to study the connection between real crime, fictional crime, and the sign systems involved in both. The objective is to argue that crime is a fertile ground for semiotic analysis, since it can provide insights not only into the cultural dimensions of crime, but also into the type of mindset that characterizes criminal acts. It is claimed that forensic semiotics would greatly enhance and broaden the scientific terrains of both forensic science and criminology.

La science du crime, ou criminologie, s'est diffusée à peu près en même temps que la littérature gothique et policière, dans la dernière partie du XIXe siècle. Une grande partie des premières techniques de détection de l'époque provenaient ou s'inspiraient de celles employées par des détectives de fiction comme C. Auguste Dupin et Sherlock Holmes. Inversement, de nouvelles techniques médico-légales ont été utilisées par les écrivains afin de rendre leurs histoires plus réalistes et conformes au scientisme culturel émergent. Le lien entre la science médico-légale et le roman policier existe encore aujourd'hui. Cette "fusion» peut être appelée sémiotique médico-légale, qui vise à étudier le lien entre le crime réel, le crime fictif et les systèmes de signes impliqués dans les deux. L'objectif est de faire valoir que le crime est un terrain fertile pour l'analyse sémiotique, car il peut fournir des indications non seulement sur les dimensions culturelles du crime, mais aussi sur le type de mentalité qui caractérise les actes criminels. On estime que la sémiotique médico-légale améliorerait et élargirait considérablement le champ scientifique de la médecine légale et de la criminologie.

\section{INDEX}

Mots-clés: sémiotique, médecine légale, roman policier, représentations médiatiques, criminologie

Keywords: semiotics, forensic science, crime stories, media representations, crime analysis

\section{AUTHORS}

\section{MARCEL DANESI}

Marcel Danesi, MSRC, est professeur de sémiotique et d'anthropologie à l'Université de Toronto (Victoria College), où il dirige les parcours de sémiotique et de théorie de la communication. Il est rédacteur en chef de Semiotica, revue de l'Association Internationale de Sémiotique. On peut citer parmi ses ouvrages récents : The Semiotics of Emoji: The Rise of Visual Language in the Age of the Internet (2016) ; Popular Culture : Introductory Perspectives, (2015, $3^{\mathrm{e}}$ édition) ; Signs of Crime : Introduction to Forensic Semiotics (2013) ; The History of the Kiss : The Birth of Popular Culture (2013); Discovery in Mathematics : An Interdisciplinary Approach (2013) ; avec Antonio Nicaso, Made Men : Mafia Culture and the Power of Symbols and Ritual (2013). 